\title{
Spatiotemporal PET Imaging of Dynamic Metabolic Changes After Therapeutic Approaches of Induced Pluripotent Stem Cells, Neuronal Stem Cells, and a Chinese Patent Medicine in Stroke
}

Hong Zhang, Fahuan Song, Caiyun Xu, Hao Liu, Zefeng Wang, Jinhui Li, Shuang Wu, YehuaShen, Yao Chen, Yunqi Zhu, Ruili Du, and Mei Tian

Department of Nuclear Medicine, The Second Affiliated Hospital of Zhejiang University School of Medicine, Hangzhou, China; Zhejiang University Medical PET Center, Zhejiang University, Hangzhou, China; Institute of Nuclear Medicine and Molecular Imaging, Zhejiang University, Hangzhou, China; Key Laboratory of Medical Molecular Imaging of Zhejiang Province, Hangzhou, China; and Collaborative Innovation Center for Brain Science, Fudan University, Shanghai, China

This study aimed to use spatiotemporal PET imaging to investigate the dynamic metabolic changes after a combined therapeutic approach of induced pluripotent stem cells (iPSCs), neuronal stem cells (NSCs), and Chinese patent medicine in a rat model of cerebral ischemia-reperfusion injury. Methods: Cerebral ischemia was established by the middle cerebral artery occlusion approach. Thirty-six male rats were randomly assigned to 1 of the 6 groups: control phosphate-buffered saline (PBS), Chinese patent medicine (Qing-kai-ling [QKL]), induced pluripotent stem cells (iPSCs), combination of iPSCs and QKL, neuronal stem cells (NSCs), and combination of NSCs and QKL. Serial ${ }^{18} \mathrm{~F}-\mathrm{FDG}$ small-animal PET imaging and neurofunctional tests were performed weekly. Autoradiographic imaging and immunohistochemical and immunofluorescent analyses were performed at 4 wk after stem cell transplantation. Results: Compared with the PBS control group, significantly higher ${ }^{18} \mathrm{~F}-\mathrm{FDG}$ accumulations in the ipsilateral cerebral infarction were observed in 5 treatment groups from weeks 1-4. Interestingly, the most intensive ${ }^{18} \mathrm{~F}-\mathrm{FDG}$ accumulation was found in the NSCs + QKL group at week 1 but in the iPSCs + QKL group at week 4. The neurofunctional scores in the 5 treatment groups were significantly higher than that of the PBS group from week 3 to 4 . In addition, there was a significant correlation between the PET imaging findings and neurofunctional recovery $(P<0.05)$ or glucose transporter-1 expression $(P<0.01)$. Immunohistochemical and immunofluorescence studies found that transplanted iPSCs survived and migrated to the ischemic region and expressed protein markers for cells of interest. Conclusion: Spatiotemporal PET imaging with ${ }^{18} \mathrm{~F}-\mathrm{FDG}$ demonstrated dynamic metabolic and functional recovery after iPSCs or NSCs combined with QKL in a rat model of cerebral ischemia-reperfusion injury. iPSCs or NSCs combined with Chinese medicine QKL seemed to be a better therapeutic approach than these stem cells used individually.

Received Jul. 2, 2015; revision accepted Aug. 31, 2015.

For correspondence or reprints contact: Mei Tian, Department of Nuclear Medicine, The Second Affiliated Hospital of Zhejiang University School of Medicine, 88 Jiefang Rd., Hangzhou, Zhejiang 310009, China.

E-mail: meitian@zju.edu.cn

Published online Sep. 10, 2015.

COPYRIGHT (c) 2015 by the Society of Nuclear Medicine and Molecular Imaging, Inc.
Key Words: induced pluripotent stem cell (iPSC); neuronal stem cell (NSC); Qing-kai-ling (QKL); positron emission tomography (PET); middle cerebral artery occlusion (MCAO)

J Nucl Med 2015; 56:1774-1779

DOI: 10.2967/jnumed.115.163170

I schemic stroke is one of the leading and life-threatening diseases, resulting in high morbidity and mortality worldwide (1). Existing therapeutic strategies including pharmacotherapy, interventional procedures, and surgery are limited in improving the neural function because of tissue damage after stroke involving multiple deleterious mechanisms (2-4). Therefore, novel therapeutic methods are needed to be explored.

Stem cell therapy is emerging as a viable option to restore neurologic function after stroke (5). Transplanted stem cells have the potential to replace the lost circuitry, produce growth or trophic factors, and stimulate the host brain cells to release these factors $(6,7)$. For instance, neuronal stem cells (NSCs) could enhance tissue repair and functional recovery after ischemic stroke because of their ability to readily differentiate into neurons, astrocytes, and oligodendrocytes both in vitro and in vivo $(8,9)$; induced pluripotent stem cells (iPSCs) can avoid the risk of immune rejection and ethical issues that hamper the clinical application. Recently, iPSCs have been applied to a variety of nervous system disease models, including ischemic stroke (10), Alzheimer disease (11), and Parkinson disease (12).

Traditional Chinese medicine is also an effective and unique approach to cure neurologic illness (especially ischemic brain injury) $(13,14)$. As a famous Chinese patent medicine, Qing-kailing (QKL) injection (modified from an-gong-niu-huang pill) has great antipyretic and antiinflammatory effects, which has been widely used for the acute stage of cerebrovascular disease in China (15). The active components of QKL are cholalic acid, deoxycholic acid, jasminoidin, and baicalin (16). A previous study has demonstrated that a mixture of cholalic acid and hyodeoxycholalic acid ( 2 active components of QKL) is able to decrease the expression 
of inflammation factors including tumor necrosis factor- $\alpha$ and interlukin-1 $\beta$ after focal cerebral ischemia (17). However, to better explore the metabolic changes and efficacy in vivo after stem cell or Chinese medicine treatment, a noninvasive, sensitive, and clinically applicable approach is warranted.

PET affords the capability to conduct both functional and molecular imaging, which can be used to monitor neurofunctional alterations in vivo (13). PET with ${ }^{18} \mathrm{~F}-\mathrm{FDG}$ has been applied to detect the subtle changes of glucose metabolism in vivo after stem cell therapy in various neurologic disease models (18).

Although stem cell transplantation or QKL treatment can promote functional recovery after middle cerebral artery occlusion (MCAO), to the best of our knowledge, no study has been published on the synergistic effect. Thus, in the present study, we use spatiotemporal PET imaging to investigate the dynamic metabolic changes after combined therapeutic approach of stem cells (iPSCs or NSCs) and Chinese patent medicine in a rat model of cerebral ischemia-reperfusion injury.

\section{MATERIALS AND METHODS}

\section{Animals and Experimental Design}

This study was approved by the Institutional Animal Care and Use Committee of Zhejiang University School of Medicine (protocol ZJU201407-1-02-066). After the MCAO procedure, 36 adult Sprague-Dawley male rats (weight, 220-270 g) were randomly assigned to 1 of the following 6 experimental groups $(n=6$ per group): phosphate-buffered saline (PBS) injection control, QKL treatment, iPSC transplantation, iPSCs combined with QKL treatment, NSC transplantation, and NSCs combined with QKL treatment. The day of MCAO was defined as day 0. Rats were administered QKL intraperitoneally daily from day 1 through 31 , and stem cells or PBS transcranial injections were performed $3 \mathrm{~d}$ after MCAO. Neurofunctional tests followed by ${ }^{18} \mathrm{~F}$-FDG small-animal PET studies were performed at day 1 (before QKL injection and stem cell transplantation) and at weeks $1,2,3$, and 4 (after stem cell transplantation). Immediately after the final ${ }^{18} \mathrm{~F}$-FDG PET scan, animals were sacrificed and brains were removed for the autoradiographic imaging, immunohistochemical staining, and immunofluorescence detection.

\section{MCAO Procedure}

Cerebral ischemia-reperfusion injury was induced by the intraluminal suture technique as previously described (19). Reperfusion was performed by withdrawal of the suture 90 min after MCAO (details are provided in the supplemental materials, available at http://jnm. snmjournals.org).

\section{Neurofunctional Test}

Animals of each group were subjected to neurofunctional testing weekly using the Garcia neurologic grading system (20) to determine whether iPSCs or NSCs combined with QKL injection could improve sensorimotor deficit after ischemic brain injury. Serial neurofunctional tests were performed and compared by a dedicated investigator who was masked to the experimental groups (details are provided in the supplemental materials).

\section{QKL Injection}

QKL injection was produced by Shenwei Pharmaceutical Co., Ltd. (lot no. 13032381), with a code number of Z13020880 approved by State Food and Drug Administration of China. Rats were administered QKL intraperitoneally at a dose of $2 \mathrm{~mL} / \mathrm{kg}$ per day for $30 \mathrm{~d}$. The dosage and frequency of administration were modified from the previous literature $(15,21)$.

\section{Cell Culture and Stem Cell Transplantation Procedures}

Green fluorescent protein (GFP)-labeled mouse iPSCs were cultured as described previously (19). Briefly, mouse iPSCs were maintained on a mitotically inactivated (mitomycin C) (Roche) mouse embryonic fibroblast feeder layer in a new type of iPSC cell culture medium. Humanized mulleri GFP (hmGFP)-labeled rat NSCs were incubated at $37^{\circ} \mathrm{C}$ in a $5 \% \mathrm{CO}_{2}$ humidified incubator in Neural Stem Cell Basal Medium (Millipore) as described previously (details are provided in the supplemental materials) (22).

The rat was placed in a stereotactic instrument (RWD Life Science Co.). A midline skin incision was made in the skull, and then a small burr hole was drilled through the skull. With a Hamilton microsyringe, $1.0 \times 10^{6}$ suspended iPSCs, NSCs, or PBS in a volume of $20 \mu \mathrm{L}$ were stereotactically injected into the right lateral ventricle (anterior-posterior, $0.9 \mathrm{~mm}$ to the bregma; mediolateral, $1.5 \mathrm{~mm}$; and dorsoventral, 3.5 $\mathrm{mm}$ from dura) in about $15 \mathrm{~min}$ (23). The needle was left in place for an additional $5 \mathrm{~min}$ and then removed slowly over $5 \mathrm{~min}$.

\section{${ }^{18}$ F-FDG Small-Animal PET Imaging and Image Analysis}

Rats were anesthetized with isoflurane $(2 \%)$ and administered approximately $18.5 \mathrm{MBq}(500 \mu \mathrm{Ci})$ of ${ }^{18} \mathrm{~F}-\mathrm{FDG}$ via the tail vein. The images were acquired in a high-resolution microPET R4 scanner (Siemens Medical Solutions) for 10-min static acquisition. ${ }^{18} \mathrm{~F}-\mathrm{FDG}$ accumulation was calculated as the percentage injected dose per gram of tissue using the AMIDE software package (version 9.2; Stanford University). To assess changes of metabolism after MCAO, regions of interest in the infarction area and the cerebellum normal area were identified in images of the transversal brain sections. The lesion-tocerebellum (L/C) ratio was used for semiquantitative analysis, which was calculated by the following formula: $\mathrm{L} / \mathrm{C}$ ratio $=$ mean counts per pixel of lesion region of interest/mean counts per pixel of cerebellum area (details are provided in the supplemental materials).

\section{Autoradiography}

To verify the image data obtained by small-animal PET, 6 animals (1 from each group) were sacrificed immediately after the final PET imaging. Coronal brain slices (1-mm thick) were cut and exposed to

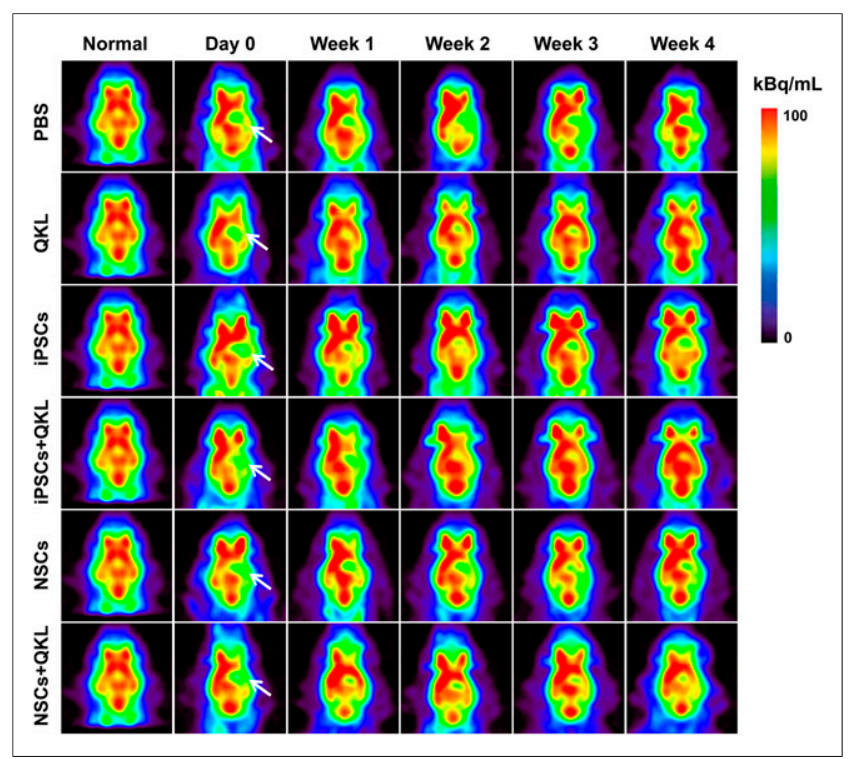

FIGURE 1. Serial representative ${ }^{18} \mathrm{~F}$-FDG PET images demonstrate metabolic recovery after each treatment in rat model of MCAO. Rat brain images are shown in axial view. Ischemic areas are indicated by white arrows. Scale was set according to signal intensity. 


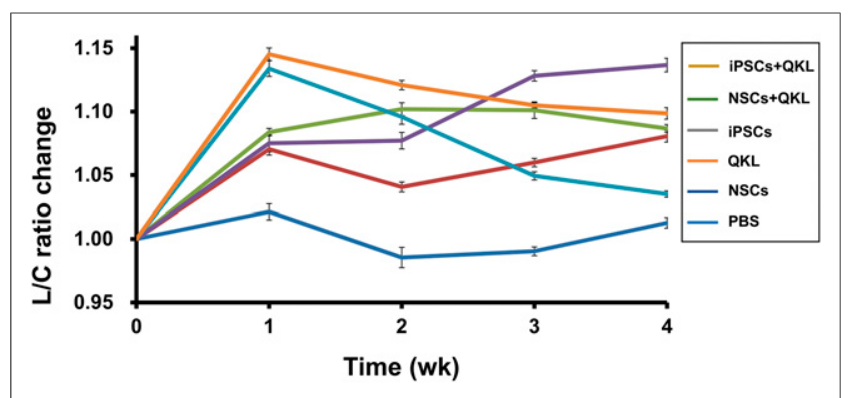

FIGURE 2. Semiquantitative analysis of glucose metabolic change in each group. Data in various groups are shown as change of L/C ratio.

an imaging plate (BAS-intraperitoneal MS 2040; Fujifilm) for 15 min, then autoradiographic images were displayed (details are provided in the supplemental materials).

\section{Immunohistochemical and Immunofluorescent Staining}

Immunohistochemical studies were performed to determine whether transplanted stem cells could induce neurogenesis or angiogenesis. Neuronal nuclei (NeuN), glial fibrillary acidic protein (GFAP), and von Willebrand factor (vWF) were used as mature neuronal, astrocyte, and endothelial cell markers, respectively, and glucose transporter (GLUT)-1 and -3 for glucose metabolic markers (Supplemental Fig. 1). The numbers of positive-stained NeuN and vWF cells were counted, and the integral optical density (IOD) of GFAP, GLUT-1, and GLUT-3 was assessed (details are provided in the supplemental materials).

To determine whether stem cells can induce neurogenesis or angiogenesis, iPSCs and NSCs were engineered to express GFP via lentiviral vectors. Double-label immunofluorescence was used to follow the fate of the GFP-labeled transplanted stem cells ( $n=1$ from each group of iPSCs, NSCs, iPSCs + QKL, and NSCs + QKL) (details are provided in the supplemental materials).

\section{Statistical Analysis}

One-way ANOVA was used to evaluate the neurofunctional score, the L/C ratio of PET, and immunohistochemically positive cells among groups. The Pearson correlation coefficient was used to analyze the correlations between ${ }^{18} \mathrm{~F}$-FDG uptake and neurologic score, IOD of GLUT-1 and IOD of GLUT-3. Data are presented as mean \pm SEM, and differences were considered significant at a $P$ value of less than 0.05 . Statistical analyses were performed with SPSS software (version 15.0; SPSS Inc.).

\section{RESULTS}

\section{iPSCs and QKL-Enhanced Glucose Metabolism and Functional Recovery After MCAO}

The ${ }^{18}$ F-FDG small-animal PET scans allowed visualization and semiquantification of the glucose metabolism throughout the brain at each time point (Fig. 1). Semiquantitative analysis of ${ }^{18}$ F-FDG radioactivity in the ischemic area showed no significant differences among the 6 groups at day 1 after MCAO (before QKL injection and stem cell transplantation). Compared with the PBS control group, ${ }^{18} \mathrm{~F}$-FDG accumulation was significantly increased in the 5 treatment groups at weeks 1 through 4, indicating that the treatments with iPSCs, NSCs, and QKL increased glucose metabolism in the cerebral ischemic area (Fig. 2). The ${ }^{18}$ F-FDG accumulations in the NSCs and NSCs + QKL groups were significantly higher than those of other groups at week $1(P<0.05)$, then decreased gradually after week 2 . Interestingly, the ${ }^{18} \mathrm{~F}-\mathrm{FDG}$ accumulation of
iPSCs + QKL presented as steadily increasing after week 2 and became significantly higher than those of the other groups at week $4(P<0.05)$. In the combination of stem cell and QKL groups (iPSCs + QKL and NSCs + QKL), ${ }^{18}$ F-FDG accumulation was higher than that of the QKL-alone group. In addition, a significant difference was found between the iPSCs and NSCs groups at week $4(P<0.05)$.

The level of ${ }^{18} \mathrm{~F}-\mathrm{FDG}$ radioactivity detected by autoradiography was similar to that assessed by ${ }^{18} \mathrm{~F}-\mathrm{FDG}$ small-animal PET in the same brain regions (Fig. 3), indicating that in vivo small-animal PET images were well consistent with in vitro autoradiographic measurements. No neuronal or cortical teratoma was observed in the transplant recipients at week 4 after iPSC and NSC transplantation.

At day 1 and week 1, no significant differences in neurologic score were detected among the 6 experimental groups (Fig. 4). The neurologic score in the NSCs group was significantly higher than those of the PBS $(P<0.01)$ and QKL $(P<0.05)$ groups at week 2. The neurologic scores in the NSCs + QKL group were significantly higher than those of the PBS group $(P<0.05)$ at week 2 . The neurologic scores in the 5 treatment groups were, respectively, higher than those of PBS control group at week 3 and week 4 . There was no significant difference in the neurologic scores among the 5 treatment groups at week 3 and week 4 . The neurologic scores of iPSCs + QKL group showed a better recovery tendency than those of the iPSCs $(P=0.094)$ and QKL $(P=0.162)$ groups at

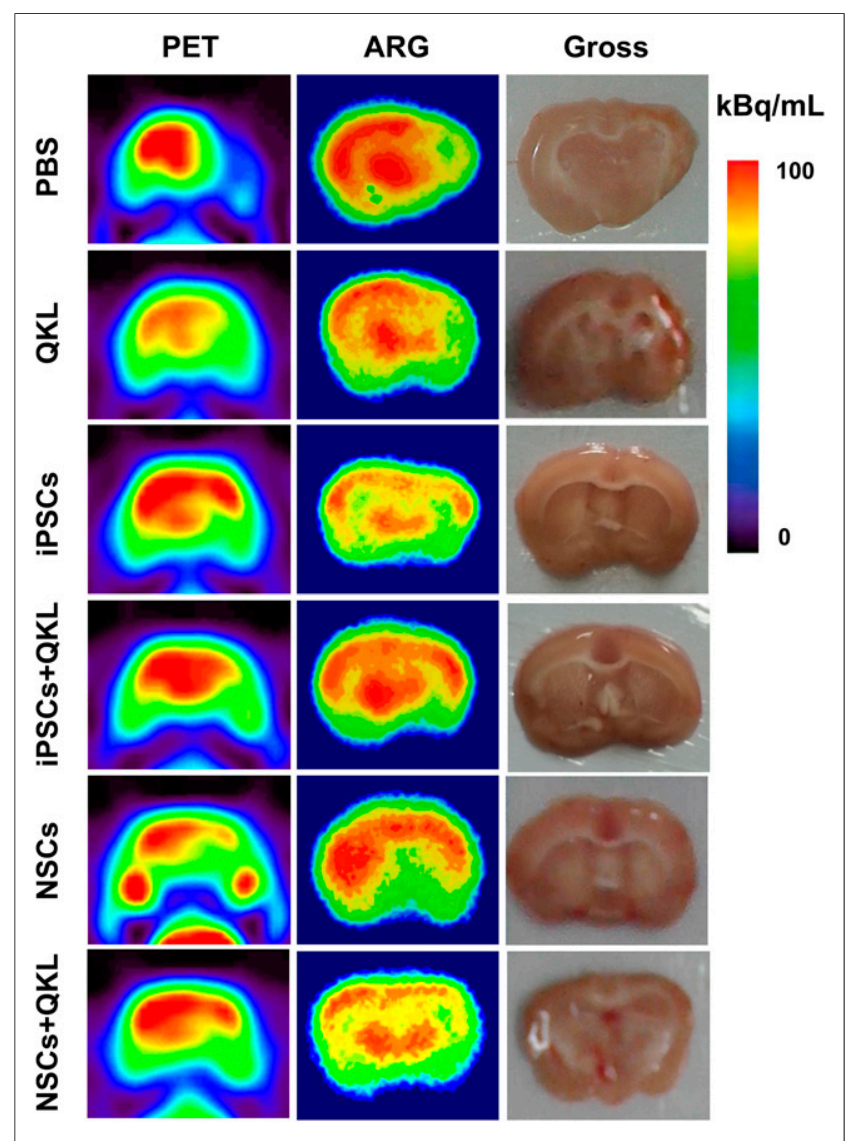

FIGURE 3. Coronal slices of small-animal PET imaging (left), autoradiographic imaging (middle), and gross sections (right). Scale was set according to signal intensity. ARG = autoradiography. 


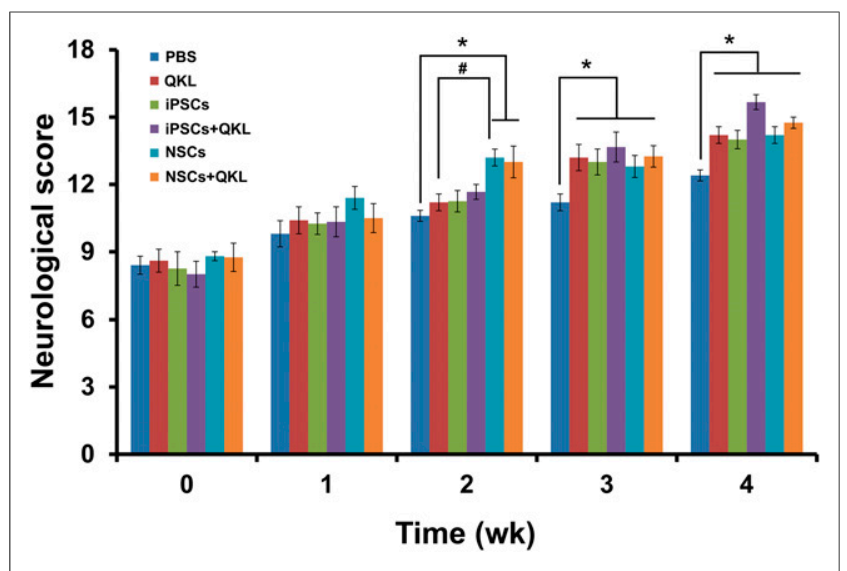

FIGURE 4. Improved neurofunction after each treatment. Groups are as follows: PBS, QKL, iPSCs, iPSCs + QKL, NSCs, and NSCs + QKL. Significant therapeutic effects were detected with use of Garcia neurologic grading system. ${ }^{\star} P<0.05$, compared with PBS group. ${ }^{*} P<0.05$, compared with NSCs group.

week 4. These results suggested that the injection of QKL and transplantation of iPSCs and NSCs improved neurologic function recovery from week 3 to 4 . Moreover, the combination of iPSCs and QKL tends to have a better promoting effect of functional recovery than iPSCs or QKL alone.

\section{iPSCs and QKL Induce Neurogenesis and Angiogenesis}

The number of NeuN-positive cells in QKL, iPSCs, iPSCs + QKL, and NSCs + QKL groups was significantly higher than that in the PBS group $(P<0.01)$. However, there was no statistically significant difference between NSCs and PBS groups $(P>0.05)$ (Fig. 5A). The IOD of GFAP in QKL, iPSCs + QKL, and NSCs + QKL groups was significantly higher than that of the PBS group $(P<0.01)$. The increased IOD of GFAP in the QKL group reached statistical significance compared with that of the iPSCs $(P=0.02)$ and NSCs $(P<0.01)$ groups. The IOD of GFAP in the iPSCs + QKL group was also significantly higher than that of iPSCs $(P<0.01)$ and NSCs $(P<0.01)$ groups (Fig. 5B). The number of vWF-positive cells in QKL, iPSCs, iPSCs + QKL, and NSCs + QKL groups was significantly higher than that in the PBS group ( $P<0.01$, respectively), but no statistically significant difference existed between the NSCs and PBS groups $(P=$ 0.10) (Fig. 5C).

The IOD of GLUT-1 in the QKL, iPSCs, and iPSCs + QKL groups was significantly higher than that in the PBS group $(P<$ 0.01 , respectively), but there was no statistically significant difference between the NSCs and PBS groups $(P>0.05)$ or the NSCs + QKL and PBS groups $(P>0.05)$. Moreover, the IOD of GLUT-1 in the NSCs group was significantly lower than that in iPSCs $(P=$ $0.01)$ and iPSCs + QKL $(P<0.01)$ groups (Fig. 5D). A significantly lower IOD of GLUT-3 was observed in the iPSCs + QKL group than in the PBS group $(P<0.01)$. The IOD of GLUT-3 in the iPSCs group also showed a lower tendency than that in the PBS group $(P=0.087)$. Similarly, the IOD of GLUT3 in iPSCs + QKL and iPSCs groups was significantly lower than that in QKL group $(P<0.01)$ and the lower IOD of GLUT-3 in the iPSCs + QKL group reached statistical significance compared with that in the NSCs group $(P<0.01)$ (Fig. 5E).

After 4 wk of therapy, tracing of the GFP-labeled cells demonstrated that the transplanted cells survived and integrated into the cerebral ischemic area (Fig. 6A). Double labeling of GFP with NeuN, GFAP, or vWF showed that the grafted iPSCs were positive for all 3 markers, suggesting that the transplanted iPSCs differentiated into mature nerve cells (neurons and astrocytes) and angiogenic cells (Figs. 6B and 6C). In addition, transplanted NSCs expressed neurons and astrocytes markers.

\section{Correlation Between PET Findings and Functional Recovery}

Glucose metabolic recovery assessed by PET imaging was significantly correlated with the number of GLUT-1-positive cells (Fig. 7A, $P<0.01$ ) and neurofunctional score (Fig. 7B, $P<0.01$ ).

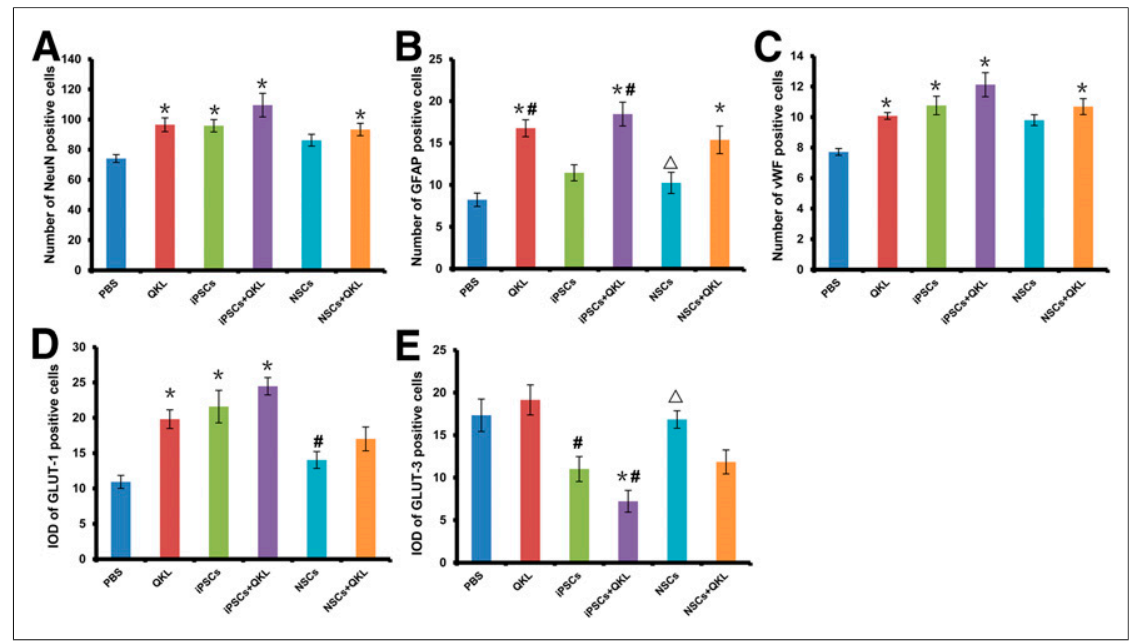

FIGURE 5. Statistical analysis of neurogenesis and metabolic markers among various treatments. (A) NeuN-positive cells. ${ }^{*} P<0.05$ vs. PBS group. (B) GFAP-positive cells. ${ }^{*} P<0.01$ vs. PBS group. ${ }^{\#} P<0.05$ vs. iPSCs group. ${ }^{\Delta} P<0.01$ vs. QKL group and iPSCs + QKL group. (C) vWF-positive cells. ${ }^{\star} P<0.05$ vs. PBS group. (D) GLUT-1-positive cells. ${ }^{\star} P<0.01$ vs. PBS group. ${ }^{\#} P<0.05$ vs. iPSCs group and iPSCs + QKL group. (E) GLUT-3-positive cells in cerebral ischemic area in each group. ${ }^{\sharp} P<0.01$ vs. QKL group. ${ }^{*} P<0.01$ vs. PBS group. ${ }^{\Delta} P<0.01$ vs. iPSCs + QKL group.

\section{DISCUSSION}

In the present study, serial ${ }^{18} \mathrm{~F}-\mathrm{FDG}$ small-animal PET scans, neurologic function tests, and immunohistochemical staining methods were used for the evaluation of therapeutic effects after stem cell and QKL injections in a rat model of MCAO. The following are the major findings. First, increased cerebral metabolism with improved neurologic function was observed after stem cell and QKL treatment (single or combined). Transplanted iPSCs survived in the infracted area and expressed the mature neuronal marker NeuN, the mature astrocyte marker GFAP, and the endothelial cell marker vWF. Transplanted NSCs also expressed mature nerve cell markers and were found in association with vWF-positive cells, suggesting the establishment of a neurovascular niche. Second, different time course patterns of glucose metabolic recovery were found 


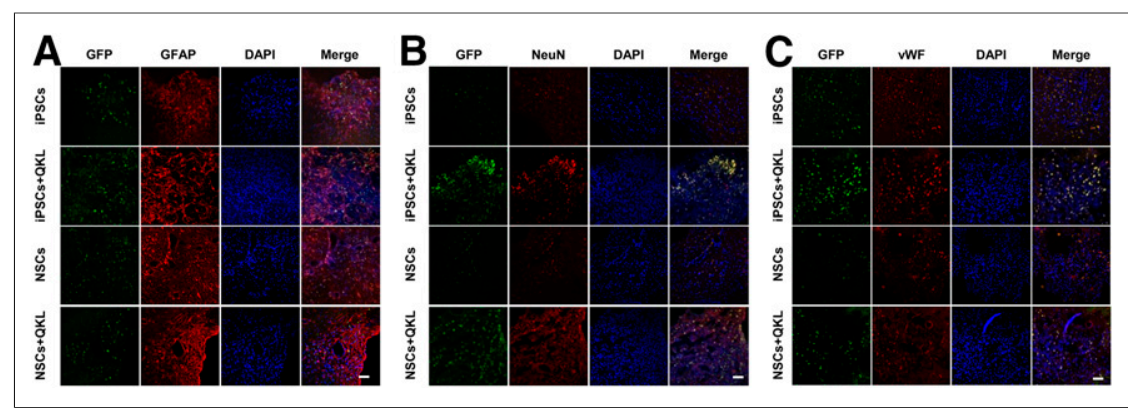

FIGURE 6. Immunofluorescent staining $(\times 200)$ of ischemic rat brains after 4-wk treatment indicated transplanted stem cells (green); antibodies against GFAP (A), NeuN (B), and VWF (C) in red; and nuclei stained with 4,6-diamino-2-phenylindole (DAPI) (blue). Merged images indicate transplanted stem cells expressing GFAP, NeuN, and vWF. Scale bar $=50 \mu \mathrm{m}$.

between NSCs and iPSCs: NSCs had a significantly better effect within the first $2 \mathrm{wk}$, whereas iPSCs had a more steady effect over $2 \mathrm{wk}$. The iPSCs + QKL group showed a steady increase of ${ }^{18} \mathrm{~F}-$ FDG accumulation and neurofunctional recovery during the 4-wk period. Last, glucose metabolic recovery assessed by PET imaging was significantly correlated with GLUT-1 expression and neurofunctional recovery. To our knowledge, this is the first study on the combination of stem cells and QKL for cerebral ischemia. By using PET imaging and the other technologies, we were able to find significant functional and metabolic improvements after stem cell and QKL therapies.

Cerebral ischemia generally involves many different deleterious processes and biochemical pathways, for instance, the production of free radicals, glutamate release, rising nitric oxide, and leukocyte

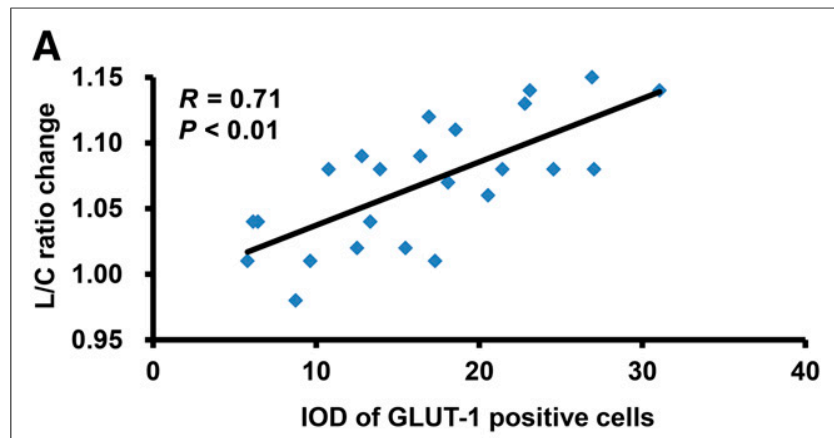

B

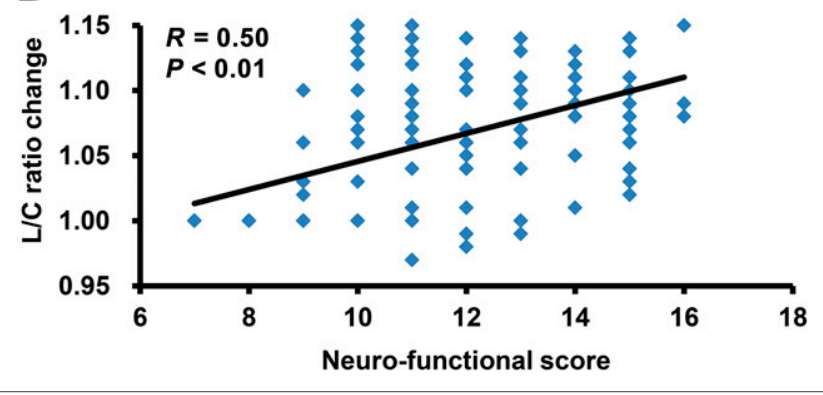

FIGURE 7. Correlation of glucose metabolic change with GLUT-1 expression and behavioral recovery. (A) IOD of GLUT-1-positive cells was significantly correlated with L/C ratio (Pearson correlation coefficient, $R=0.71, P<0.01, n=24$ ). (B) Neurologic scores were significantly correlated with L/C ratio change (Pearson correlation coefficient, $R=$ $0.50, P<0.01, n=130$ ). infiltration, which eventually lead to cellular injury and death after reperfusion (24,25). In our study, significant positive effects were found after stem cell combined with QKL administration. Immunohistochemical and immunofluorescence staining verified that iPSCs and NSCs migrated to the area close to the ischemic area, where they expressed the mature neuronal marker NeuN, mature astrocyte marker GFAP, and endothelial cell marker vWF. These findings corroborate other previous studies (on NSCs or iPSCs used individually), which demonstrated that stem cells survived, migrated, and differentiated into mature neurons (26) or angiogenic cells (19) and improved functional recovery via promoting vascular endothelial growth factor expression and enhancing endogenous plasticity in the injured brain (27). Transplantation of iPSCs could improve the motor function, reduce infarct size, attenuate inflammation cytokines, and mediate neuroprotection after ischemic stroke $(10,28)$. Furthermore, QKL adjuvant treatment has been found to alleviate the aggravated inflammatory reaction and protected injured brain by decreasing the levels of $\mathrm{Ca}^{2+}$ and matrix metalloproteinase-9 (29). Active components of QKL were able to protect the neuron through inhibiting the level of tumor necrosis factor- $\alpha$ and interlukin- $1 \beta$. When these are taken together, significant recovery after stem cells combined with QKL might due to a favorable microenvironment for both endogenous and exogenous cells.

The second important finding of this study was the patterns of glucose metabolic recovery after iPSCs and NSCs (with or without QKL injection). NSCs had a significantly better effect within the first $2 \mathrm{wk}$, whereas iPSCs had a more steady effect over $2 \mathrm{wk}$. This observation was not entirely unexpected as it has been found that iPSCs could form functional neurons and improve the neurologic function up to 4-12 wk $(19,27)$, whereas NSCs have significant therapeutic effects at $2 \mathrm{wk}$ after transplantation $(30,31)$, yet the prolonged effect in vivo has not been universally demonstrated. We suppose that cerebral ischemia appeared to activate the neurogenesis program: transplanted NSCs may act as a functional cell type in an earlier time course after transplantation, whereas iPSCs might differentiate into a more specific functional cell type before promoting the recovery of cerebral ischemia.

Previous studies addressing brain glucose utilization have considered GLUT-1 is predominantly localized on the endothelial cells, and GLUT-3 is present in the neurons $(32,33)$. In this present study, glucose metabolic recovery assessed by PET imaging was significantly correlated with GLUT-1 expression and neurofunctional recovery. Especially, the overexpression of GLUT-1 (in the groups of QKL, iPSCs, iPSCs + QKL, and NSCs + QKL) was consistent with our findings on ${ }^{18} \mathrm{~F}$-FDG PET imaging and immunostaining of $\mathrm{vWF}$, which indicated the delivery of glucose from blood to brain through the blood-brain barrier by GLUT-1. Similar results of GLUT-1 overexpression were obtained in a rat model of cerebral ischemia after combined treatment of bone marrow stromal cells and sodium ferulate (a drug used in traditional Chinese medicine for treatment of cerebro- or cardiovascular disease and to prevent thrombosis) (34). More interestingly, we observed a significantly lower expression of GLUT-3 in the iPSCs and iPSCs + QKL groups than the PBS, QKL, NSCs, and NSCs + QKL groups. Our consideration is that, under the microenvironment 
with iPSCs, the transportation of glucose into neurons may not be mediated by GLUT-3. This could be explained partially by a recent study on a $\mathrm{Na}^{+}$-glucose cotransporter (SGLT)-specific molecular imaging probe, $\alpha$-methyl-4-deoxy-4- ${ }^{18} \mathrm{~F}$-fluoro-D-glucopyranoside, in a rat model of blood-brain barrier disruption, in which the expression of SGLT1 protein was found mainly in neuron cell bodies, exons, and dendrites (33).

In summary, our study is the first to use a combination of stem cells and Chinese medicine for stroke therapy and report beneficial effects of the combination therapy. In the future, this study should be extended beyond the 4-wk treatment period to monitor the long-term therapeutic response after iPSC and NSC transplantation with or without QKL. This is especially important in view of the studies reporting teratogenic potential of stem cells in rodents' postischemic brain, although we did not detect tumor formation in any of the animals during the $4 \mathrm{wk}$ of treatment.

\section{CONCLUSION}

Spatiotemporal PET imaging with ${ }^{18}$ F-FDG demonstrated dynamic metabolic and functional recovery after iPSCs or NSCs combined with QKL in a rat model of cerebral ischemia-reperfusion injury. iPSCs or NSCs combined with Chinese medicine QKL seemed to be a better therapeutic approach than these cells used individually.

\section{DISCLOSURE}

The costs of publication of this article were defrayed in part by the payment of page charges. Therefore, and solely to indicate this fact, this article is hereby marked "advertisement" in accordance with 18 USC section 1734 . This work was partly sponsored by grants from the National Science Foundation of China (NSFC) (no. 81271601, 81425015), Ministry of Science and Technology of China (2011CB504400, 2015DFG32740), Health Bureau of Zhejiang Province (WKJ2013-2-016), Specialized Research Fund for the Doctoral Program of Higher Education (20130101110015), and Zhejiang Provincial Natural Science Foundation of China (LR13H180001). No other potential conflict of interest relevant to this article was reported.

\section{ACKNOWLEDGMENT}

We thank Dr. Weizhong $\mathrm{Gu}$ for his excellent technical assistance and valuable advice on immunohistochemistry and immunofluorescence.

\section{REFERENCES}

1. Feigin VL, Forouzanfar MH, Krishnamurthi R, et al. Global and regional burden of stroke during 1990-2010: findings from the Global Burden of Disease Study 2010. Lancet. 2014;383:245-254.

2. Fisher M. New approaches to neuroprotective drug development. Stroke. 2011;42: $\mathrm{S} 24-\mathrm{S} 27$.

3. Lo EH, Dalkara T, Moskowitz MA. Mechanisms, challenges and opportunities in stroke. Nat Rev Neurosci. 2003;4:399-415.

4. Langhorne P, Bernhardt J, Kwakkel G. Stroke rehabilitation. Lancet. 2011;377: 1693-1702.

5. Kalladka D, Muir KW. Brain repair: cell therapy in stroke. Stem Cells Cloning. 2014;7:31-44

6. Liu X, Ye R, Yan T, et al. Cell based therapies for ischemic stroke: from basic science to bedside. Prog Neurobiol. 2014;115:92-115.
7. Daadi MM, Hu S, Klausner J, et al. Imaging neural stem cell graft-induced structural repair in stroke. Cell Transplant. 2013;22:881-892.

8. Gage FH. Mammalian neural stem cells. Science. 2000;287:1433-1438.

9. Zhang $\mathrm{H}$, Zheng $\mathrm{X}$, Yang $\mathrm{X}$, et al. ${ }^{11} \mathrm{C}-\mathrm{NMSP} /{ }^{18} \mathrm{~F}-\mathrm{FDG}$ microPET to monitor neural stem cell transplantation in a rat model of traumatic brain injury. Eur $J$ Nucl Med Mol Imaging. 2008;35:1699-1708.

10. Chen SJ, Chang CM, Tsai SK, et al. Functional improvement of focal cerebral ischemia injury by subdural transplantation of induced pluripotent stem cells with fibrin glue. Stem Cells Dev. 2010;19:1757-1767.

11. Fan X, Sun D, Tang X, Cai Y, Yin ZQ, Xu H. Stem-cell challenges in the treatment of Alzheimer's disease: a long way from bench to bedside. Med Res Rev. 2014;34:957-978.

12. Roessler R, Boddeke E, Copray S. Induced pluripotent stem cell technology and direct conversion: new possibilities to study and treat Parkinson's disease. Stem Cell Rev. 2013;9:505-513.

13. Heiss WD. Radionuclide imaging in ischemic stroke. J Nucl Med. 2014;55:1831-1841.

14. Wang Z, Wan H, Li J, Zhang H, Tian M. Molecular imaging in traditional Chinese medicine therapy for neurological diseases. Biomed Res Int. 2013;2013:608430.

15. Cheng F, Zhong X, Lu Y, et al. Refined Qingkailing protects MCAO mice from endoplasmic reticulum stress-induced apoptosis with a broad time window. Evid Based Complement Alternat Med. 2012;2012:567872.

16. Hua Q, Zhu X, Li P, et al. Refined Qing Kai Ling, traditional Chinese medicinal preparation, reduces ischemic stroke-induced infarct size and neurological deficits and increases expression of endothelial nitric oxide synthase. Biol Pharm Bull. 2008;31:633-637.

17. Hua Q, Zhu XL, Li PT, et al. The inhibitory effects of cholalic acid and hyodeoxycholalic acid on the expression of TNF- $\alpha$ and IL-1 $\beta$ after cerebral ischemia in rats. Arch Pharm Res. 2009;32:65-73.

18. Wang J, Tian M, Zhang H. PET molecular imaging in stem cell therapy for neurological diseases. Eur J Nucl Med Mol Imaging. 2011;38:1926-1938.

19. Wang J, Chao F, Han F, et al. PET demonstrates functional recovery after transplantation of induced pluripotent stem cells in a rat model of cerebral ischemic injury. J Nucl Med. 2013;54:785-792.

20. Garcia JH, Wagner S, Liu KF, Hu XJ. Neurological deficit and extent of neuronal necrosis attributable to middle cerebral artery occlusion in rats: statistical validation. Stroke. 1995;26:627-634.

21. Lv L, Liu Y, Shi HF, Dong Q. Qingkailing injection attenuates apoptosis and neurologic deficits in a rat model of intracerebral hemorrhage. J Ethnopharmacol. 2009;125:269-273.

22. Palmer TD, Markakis EA, Willhoite AR, Safar F, Gage FH. Fibroblast growth factor-2 activates a latent neurogenic program in neural stem cells from diverse regions of the adult CNS. J Neurosci. 1999;19:8487-8497.

23. Sugiura S, Kitagawa K, Tanaka S, et al. Adenovirus-mediated gene transfer of heparin-binding epidermal growth factor-like growth factor enhances neurogenesis and angiogenesis after focal cerebral ischemia in rats. Stroke. 2005;36:859-864.

24. Green AR, Shuaib A. Therapeutic strategies for the treatment of stroke. Drug Discov Today. 2006;11:681-693.

25. Woodruff TM, Thundyil J, Tang SC, Sobey CG, Taylor SM, Arumugam TV. Pathophysiology, treatment, and animal and cellular models of human ischemic stroke. Mol Neurodegener. 2011;6:11.

26. Rosenblum S, Wang N, Smith TN, et al. Timing of intra-arterial neural stem cell transplantation after hypoxia-ischemia influences cell engraftment, survival, and differentiation. Stroke. 2012;43:1624-1631.

27. Oki K, Tatarishvili J, Wood J, et al. Human-induced pluripotent stem cells form functional neurons and improve recovery after grafting in stroke-damaged brain. Stem Cells. 2012;30:1120-1133.

28. Miljan EA, Sinden JD. Stem cell treatment of ischemic brain injury. Curr Opin Mol Ther. 2009;11:394-403.

29. Yue S, Li Q, Liu S, et al. Mechanism of neuroprotective effect induced by QingKaiLing as an adjuvant drug in rabbits with E. coli bacterial meningitis. Acta Neurochir Suppl. 2006;96:413-418.

30. Tang Y, Wang J, Lin X, et al. Neural stem cell protects aged rat brain from ischemia-reperfusion injury through neurogenesis and angiogenesis. J Cereb Blood Flow Metab. 2014;34:1138-1147.

31. Guzman R, De Los Angeles A, Cheshier S, et al. Intracarotid injection of fluorescence activated cell-sorted CD49d-positive neural stem cells improves targeted cell delivery and behavior after stroke in a mouse stroke model. Stroke. 2008;39:1300-1306.

32. Duelli R, Kuschinsky W. Brain glucose transporters: relationship to local energy demand. News Physiol Sci. 2001;16:71-76.

33. Yu AS, Hirayama BA, Timbol G, et al. Regional distribution of SGLT activity in rat brain in vivo. Am J Physiol Cell Physiol. 2013;304:C240-C247.

34. Zhao Y, Lai W, Xu Y, Li L, Chen Z, Wu W. Exogenous and endogenous therapeutic effects of combination Sodium Ferulate and bone marrow stromal cells (BMSCs) treatment enhance neurogenesis after rat focal cerebral ischemia. Metab Brain Dis. 2013;28:655-666. 